\title{
A Versatile AuNP Synthetic Platform for Decoupled Control of Size and Surface Composition
}

\author{
Ye Yang, ${ }^{\circledR}$ Luis A. Serrano, ${ }^{\circledR}$ and Stefan Guldin* \\ Department of Chemical Engineering, University College London, Torrington Place, London WC1E 7JE, U.K.
}

Supporting Information

ABSTRACT: While a plethora of protocols exist for the synthesis of sub-10-nm gold nanoparticles (AuNPs), independent control over the size and surface composition remains restricted. This poses a particular challenge for systematic studies of AuNP structurefunction relationships and the optimization of crucial design parameters. To this end, we report on a modular two-step approach based on the synthesis of AuNPs in oleylamine (OAm) followed by subsequent functionalization with thiol ligands and mixtures thereof.

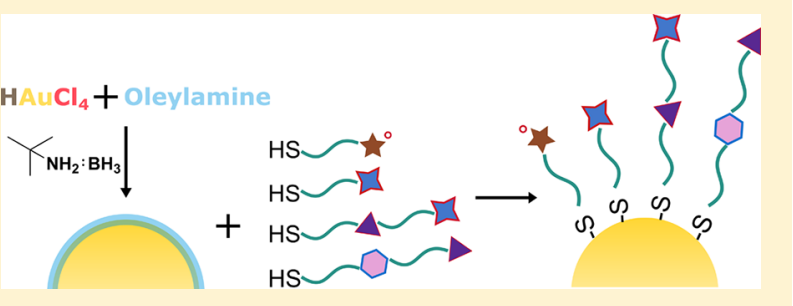
The synthesis of OAm-capped AuNPs enables fine-tuning of the core size in the range of $2-7$ nm by varying the reaction temperature. The subsequent thiol-for-OAm ligand exchange allows a reliable generation of thiol-capped AuNPs with target surface functionality. The compatibility of this approach with a vast library of thiol ligands provides detailed control of the mixed ligand composition and solubility in a wide range of solvents ranging from water to hexane. This decoupled control over the AuNP core and ligand shell provides a powerful toolbox for the methodical screening of optimal design parameters and facile preparation of AuNPs with target properties.

\section{INTRODUCTION}

Among all of the nanomaterial systems, gold nanoparticles (AuNPs) are one of the most widely studied material platforms. With distinct chemical and physical properties, AuNPs have become vital components in various optical, ${ }^{1,2}$ catalytic, ${ }^{3-5}$ chemical, and biomedical $^{6-9}$ applications. Sub-10-nm AuNPs are of particular interest for a number of reasons. Functionalization with a densely packed self-assembled monolayer of thiol ligands offers reliable core protection and long-term colloidal stability, provided by the strong interaction between gold and sulfur. ${ }^{10,11}$ The broad library of available capping agents and its size range makes these types of AuNPs promising candidates as biomedical agents and as building blocks in constructing nanostructures with higher orders. ${ }^{12-15}$

The chemical nature of the stabilizing ligand shell structure is decisive for many NP properties, including solubility, chemical reactivity, and binding affinity. ${ }^{16-20}$ It has been reported that the interrelationship between NP size and surface properties is key for controlling cellular uptake ${ }^{21}$ and protein adsorption. ${ }^{22,23}$ This demand poses challenges for existing preparation protocols as most are parameter-sensitive wet-chemistry syntheses. For example, although uniformly distributed thiolprotected AuNPs can be synthesized, both the classical onestep Brust-Schiffrin method ${ }^{24}$ and the one-phase version ${ }^{25}$ suffer from a divergence of the NP core diameter when varying the composition of the ligand shell, making comparability and systematic studies difficult. ${ }^{26}$

Exchange of the capping agent offers a viable route to decoupling gold core preparation from ligand shell functionalization. ${ }^{27,28}$ In the so-called place-exchange reaction, an excess of target ligands is introduced to replace the existing protecting shell. $^{29}$ The kinetics and evolution of the ligand shell morphology in thiol-for-thiol exchange reactions were recently studied in great detail. ${ }^{30}$ An alternative is the synthesis of AuNPs with a more labile intermediate capping agent to facilitate the exchange. The use of AuNP protectants such as citrate $^{31}$ phosphine, ${ }^{32,33}$ 4-(N,N-dimethylamino)pyridine (DMAP), ${ }^{34,35}$ dioctylamine (DOA), ${ }^{36}$ and tetraoctylammonium bromide $(\mathrm{TOAB})^{37}$ allows us to separate the synthesis of the AuNP core and the formation of a tailored ligand shell via a thiol-for-agent ligand-exchange procedure. ${ }^{37}$

While each of the routes described above has been successfully implemented in certain protocols, their general applicability is limited by drawbacks such as (i) the available AuNP size range and size tunability, (ii) the uniformity in size and shape of the AuNP core, (iii) the stability and exchangeability of the intermediate capping agent, (iv) the yield and scalability of the reaction, and (v) the practicality of the procedure. Reliable thiol-for-thiol exchange is timeconsuming, with most protocols requiring daylong procedures. ${ }^{30}$ Because of the strong association between the capping agent and the gold core, it is generally challenging to achieve complete and homogeneous substitution. Typically, replacement thiols are introduced in large excess, which raises concerns over partial etching of the AuNP core. ${ }^{38,39}$ For citrate-based AuNPs, the available size range is typically 10$150 \mathrm{~nm},{ }^{40}$ though some protocols exists to tune the mean size below this range. ${ }^{41}$ In contrast, phosphine-based protocols are

Received: February 1, 2018

Revised: April 13, 2018

Published: May 16, 2018 
limited to $<2 \mathrm{~nm}$. For DMAP-based, DOA-based, and TOABbased AuNPs, the available uniformity and tunability of the AuNP core are somewhat restricted. ${ }^{36,42,43}$ Moreover, it remains a challenge to achieve a satisfiable yield and compatibility with large-scale synthesis when the intermediate AuNP complex is unstable. ${ }^{33,44}$

The use of oleylamine (OAm) in AuNP synthesis has attracted considerable interest due to its ability to play multiple roles as surfactant, solvent, and reducing agent. ${ }^{45}$ OAm alone is able to reduce the gold precusor and stabilize $>8 \mathrm{~nm}$ AuNPs at elevated temperature $\left(>80{ }^{\circ} \mathrm{C}\right)$ in small-scale syntheses. ${ }^{46-49}$ Recently, the introduction of additional reducing agents enabled the facile production of smaller AuNPs with a narrow size distribution and size tunability near room temperature..$^{50,51}$ The potential of further surface functionalization by ligand exchange was recently validated for alkylthiol, ${ }^{52}$ biphenylthiol, ${ }^{53}$ azothiol, $^{54}$ and charged thiol mixtures. ${ }^{55}$

Based on OAm as intermediated capping agent, we herein report on a two-step protocol for independent control over AuNP core and ligand shells. We study the applicability of this approach to produce thiol-capped AuNPs via thiol-for-OAm ligand replacement and establish a successful procedure to tune the core size as well as the capping-layer composition within a broad library of thiol ligands and mixtures thereof. The resulting AuNPs exhibit solubilities ranging from hexane to water and are ideally suited for systematic studies of AuNP structure-function relationships as well as AuNP engineering of crucial design parameters.

\section{EXPERIMENTAL SECTION}

Synthesis of OAm-AuNPs. The chemicals were purchased from Sigma-Aldrich (U.K.) and used without further purification unless otherwise stated. A generic OAm-AuNP synthesis started with the preparation of the precursor at room temperature $\left(\mathrm{RT}, 20{ }^{\circ} \mathrm{C}\right.$ ) by dissolving $0.5 \mathrm{mmol}$ of hydrogen tetrachloroaurate (III) hydrate $\left(\mathrm{HAuCl}_{4} \cdot 3 \mathrm{H}_{2} \mathrm{O}, 95 \%\right)$ in a $40 \mathrm{~mL}$ solvent mixture of OAm (C18 content: $80 \%-90 \%)$ and $n$-octane $(97 \%)(1: 1 \mathrm{v} / \mathrm{v})$. The solution was sonicated under a flow of argon (Ar) for $10 \mathrm{~min}$ before stirring at a designated reaction temperature. For the precise control of the reaction temperature, a $100 \mathrm{~mL}$ jacketed flask was used in the synthesis with a temperature-controlled circulating bath (Grant Instruments, GR150-R2). The reducing solution was prepared by dissolving 0.5 mmol of $t$-butylamine-borane complex (tBAB, 97\%) in $1 \mathrm{~mL}$ of OAm and $1 \mathrm{~mL}$ of octane before the injection into the precursor solution under vigorous stirring. The mixture was left reacting in an $\mathrm{Ar}$ atmosphere at the specified reaction temperature for $2 \mathrm{~h}$ before $30 \mathrm{~mL}$ of acetone was added to quench the reaction. Various batches of OAm-AuNPs were prepared in octane to study the effect of the reaction temperature in the range of $1-50{ }^{\circ} \mathrm{C}$. The scalability of this method was studied at $15{ }^{\circ} \mathrm{C}$, when 0.25 and $1.0 \mathrm{mmol}$ of $\mathrm{HAuCl}_{4}$ were used, respectively. The AuNPs were collected by centrifugation at $5000 \mathrm{~g}$ for $10 \mathrm{~min}$ and then redispersed in dichloromethane (DCM). The obtained samples were dried in a vacuum desiccator after repeated washing in ethanol.

Thiol-for-OAm Ligand Exchange for AuNPs with a Homoligand Shell. The general procedure of the ligand-exchange process started by dissolving $0.2 \mathrm{mmol}$ of the designated thiol ligand mixture in $10 \mathrm{~mL}$ of DCM by vigorous stirring at RT for $10 \mathrm{~min}$. Subsequently, $30 \mathrm{mg}$ of the OAm-capped AuNPs in $5 \mathrm{~mL}$ of DCM solution was injected, and the solution was allowed to react at RT for $6 \mathrm{~h}$. To terminate the ligand-exchange procedure of organic-soluble AuNPs, the solution was first evaporated in a rotary evaporator before washing with methanol $(\mathrm{MeOH})$ or acetone three times to remove the excess free ligands in solution. In the case of targeting water-soluble AuNPs, $\mathrm{MeOH}$ or acetone was directly added to terminate the reaction. The functionalized AuNPs were collected by repetitive centrifugation at
$5000 \mathrm{~g}$ for $10 \mathrm{~min}$ before further drying in a vacuum desiccator. Various batches using the following thiol ligands were prepared with the same protocol: 1-hexanethiol (HT, 97\%), 1-octanethiol (OT, 98\%), 11mercapto-1-undecanol (MUO, 97\%), 2-phenylethanethiol (PET, $98 \%$ ), MDDCBO (synthesis adapted from a reported protocol, ${ }^{42}$ see SI), MNT (synthesis adapted from protocol, ${ }^{56}$ see SI), 11-mercaptoundecanoic acid (MUA, 95\%), and 11-mercapto-1-undecanesulfonate (MUS) (synthesized as reported, ${ }^{57}$ see SI). For a more detailed summary of the results, see Table 1 .

Table 1. Thiol Ligands Used for Homoligand AuNPs

$\begin{array}{ll}\text { Ligand } & \begin{array}{l}\text { Resulting AuNP solubility } \\ \text { Toluene, DCM, Hexane, Chloroform } \\ \mathrm{MeOH}, \mathrm{EtOH}\end{array} \\ \mathrm{MUS} & \end{array}$

To compare the ligand density of thiol-protected AuNPs prepared by the two-step OAm ligand-exchange method via a classical one-step synthesis, OT-capped AuNPs were prepared following the single-phase protocol developed by Stucky and co-workers. ${ }^{25}$ In detail, $0.5 \mathrm{mmol}$ of chloro(triphenylphosphine)gold(I) (95\%) was mixed with $2 \mathrm{mmol}$ of $\mathrm{OT}$ at $80^{\circ} \mathrm{C}$ followed by subsequent reduction with $5 \mathrm{mmol}$ of $\mathrm{tBAB}$ at the same temperature for $1 \mathrm{~h}$. The solution was cooled to $40{ }^{\circ} \mathrm{C}$ before the addition of $30 \mathrm{~mL}$ of $\mathrm{MeOH}$. The AuNPs were collected after washing three times in acetone and subsequent vacuum drying.

Thiol-for-OAm Ligand Exchange for AuNPs with a MixedLigand Shell. Similar to the functionalization of a homoligand shell, mixed-ligand AuNPs were prepared by mixing $0.2 \mathrm{mmol}$ of the thiol ligand mixture at a given feed ratio with $30 \mathrm{mg}$ of the OAm-capped AuNPs in DCM. Functionalized AuNPs with the following ligand mixtures (molar feed ratio) were used: MUO-PET (50-50\%), MNTHT (60-40\%), MDDCBO-HT (60-40\%), and MUS-OT (60-40\%). For MUS-OT composition variation, the following prescriptions were introduced: $80-20,60-40,40-60$, and $20-80 \%$.

Characterization. A high-resolution $200 \mathrm{keV}$ transmission electron microscope (TEM) system (JEOL, JEM-2010) was used for size imaging. UV-visible (UV-vis) absorption was measured with a fiber-based setup that combined a stabilized tungsten-halogen light source (Thorlabs, SLS201/M, a cuvette holder (Quantum Northwest, qpod 2e) and a high-sensitivity spectrometer (Ocean Optics, QE-Pro). Thermogravimetric analysis (TGA) was studied with a thermogravimetric analyzer (TA Instruments, TGA 550) at a ramp rate of $20^{\circ} \mathrm{C} /$ min from RT to $550{ }^{\circ} \mathrm{C}$. Proton nuclear magnetic resonance $\left({ }^{1} \mathrm{H}\right.$ NMR) results were obtained at $400 \mathrm{MHz}$ (Bruker, Avance III). NMR analysis was carried out in two steps. First, the effectiveness of AuNP washing was verified by line broadening of the surface-bound thiol ligands. Subsequently, ligand desorption was induced by the addition of iodine $\left(\mathrm{I}_{2}\right)$; see the Supporting Information.

\section{RESULTS AND DISCUSSION}

The synthesis platform based on AuNP synthesis with OAm as an intermediate capping agent and subsequent thiol-for-OAm ligand exchange is illustrated in Figure 1. While the reaction temperature is used as sole parameter to tune the AuNP core size in the initial synthesis, subsequent ligand exchange involving thiols (in minimum excess) allows us to produce thiol-capped AuNPs with ionic, amphiphathic, and hydrophobic ligand functionalization as well as mixtures thereof. 


\section{STEP 2: Thiol-for-OAm ligand exchange}

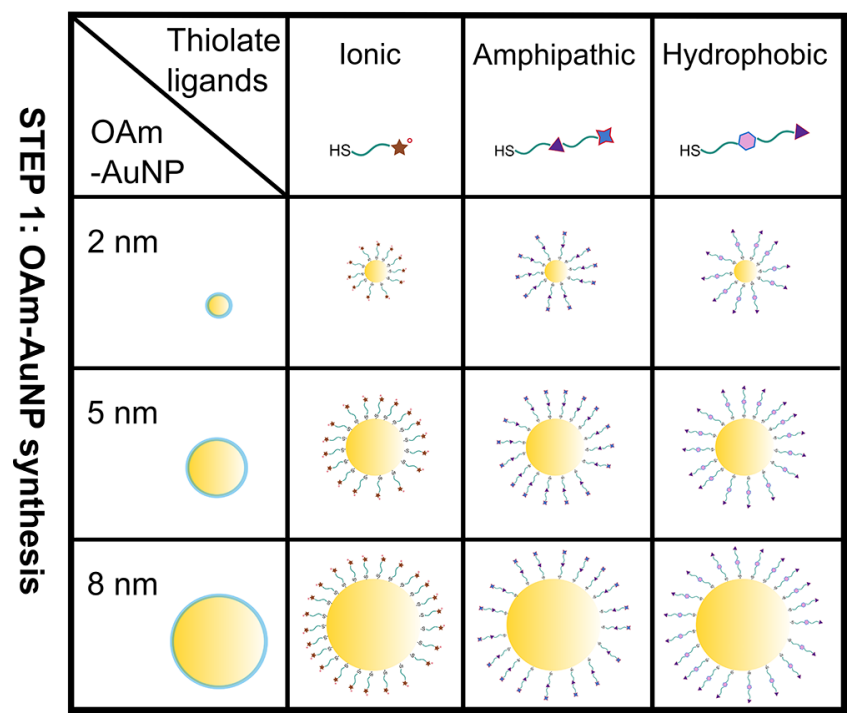

Figure 1. Schematic of two-step gold nanoparticle (AuNP) preparation consisting of AuNP synthesis with oleylamine (OAm) and subsequent functionalization via thiol-for-OAm ligand exchange.

Fine Tuning of the OAm-AuNP Core Size with Temperature. The synthesis of OAm-capped AuNPs was revisited using the burst nucleation method based on the reduction of $\mathrm{HAuCl}_{4}$ by $\mathrm{HBAB}^{50} \mathrm{~A}$ representative image of AuNPs stemming from synthesis at different reaction temperatures is shown in Figure 2a. The resulting size distribution as a function of reaction temperature is summarized in Figure $2 b$ with characteristic histograms for each temperature in the Supporting Information (Figure S1). A clear effect of the reaction temperature variation on the resulting AuNP size can be observed. For example, at a reaction temperature of $1{ }^{\circ} \mathrm{C}$, AuNPs with an average size of $6.3 \pm 0.7 \mathrm{~nm}$ were obtained, which reduced to $5.0 \pm 0.6 \mathrm{~nm}$ for $10^{\circ} \mathrm{C}, 4.0 \pm 0.6 \mathrm{~nm}$ for 20 ${ }^{\circ} \mathrm{C}, 3.0 \pm 0.5 \mathrm{~nm}$ for $30{ }^{\circ} \mathrm{C}$, and $2.2 \pm 0.4 \mathrm{~nm}$ for $40^{\circ} \mathrm{C}$. An increase in the reaction temperature to $50{ }^{\circ} \mathrm{C}$ did not lead to a further reduction of the average diameter $(2.2 \pm 0.4 \mathrm{~nm})$. Thus, by controlling only a single experimental parameter, the reaction temperature, the average size of the AuNPs was systematically tuned in the range of $2-7 \mathrm{~nm}$. These results are in line with reported values for OAm-based synthesis in octane $^{26}$ and show a trend similar to that of the OAm-based synthesis in tetralin. ${ }^{50}$ Moreover, this approach yielded excellent scalability as evidenced by the consistent results when varying the batch size by a factor of 4 . At $15{ }^{\circ} \mathrm{C}$, the size distributions for $0.25,0.5$, and $1.0 \mathrm{mmol} \mathrm{HAuCl}_{4}$ batch sizes were determined to be $4.6 \pm 0.5,4.6 \pm 0.6$, and $4.7 \pm 0.6 \mathrm{~nm}$, respectively (Supporting Information, Figure S2). This simple tunability over such a broad size range makes the OAm-based AuNP synthesis an ideal base for the subsequent functionalization via thiol-for-OAm replacement.

Ligand Packing Density. While OAm-based synthesis was previously introduced as a reliable procedure for AuNP preparation, the primary aim of this work was to use this protocol as a base for subsequent functionalization with a broad range of target thiol ligands and mixtures thereof. In order to compare the ligand shell of 1-octanethiol (OT)-capped AuNPs after thiol-for-OAm exchange (OAm-OT AuNP) with those obtained directly by a one-phase method (OT AuNP), batches (a)
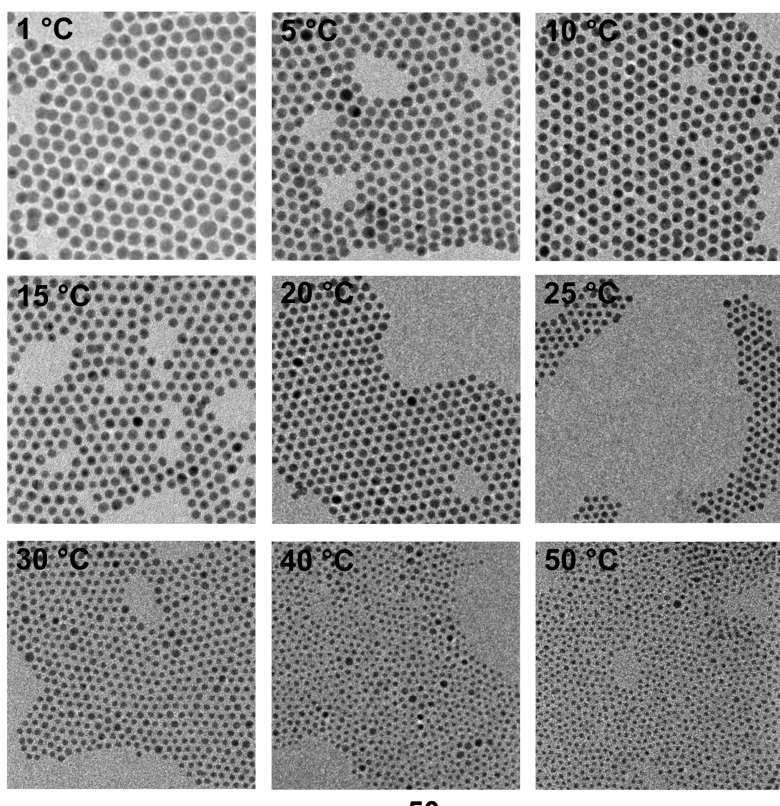

$50 \mathrm{~nm}$

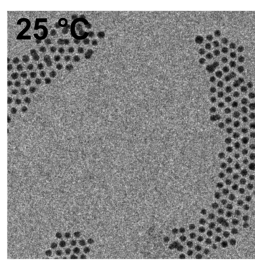

(b)

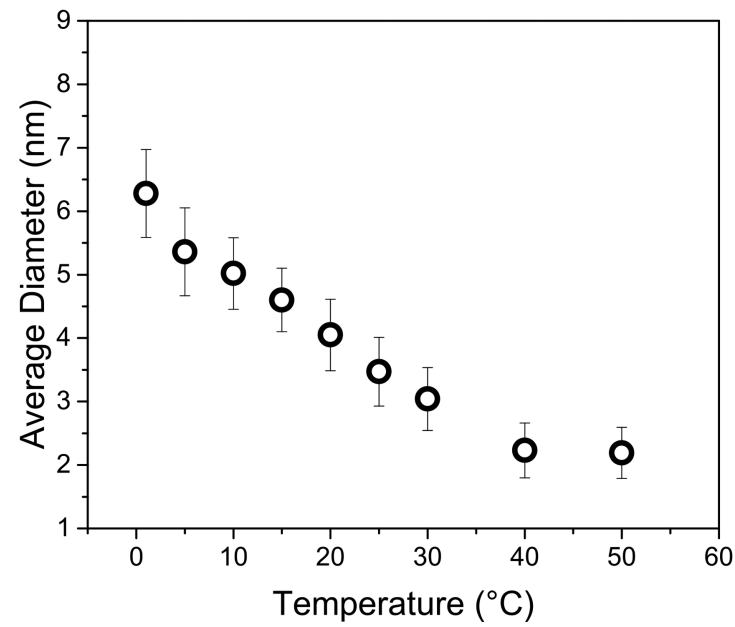

Figure 2. Oleylamine-based synthesis of gold nanoparticles. (a) Representative TEM images for different reaction temperatures. (b) Average size and standard deviation of the nanoparticle populations as a function of reaction temperature.

were prepared for each method and dried thoroughly by rotary evaporation and subsequently overnight in a vacuum desiccator. The TGA results of those samples are presented in Figure S8. The weight loss observed in the range below $600{ }^{\circ} \mathrm{C}$ can be exclusively related to the decomposition of the organic ligand shell as most of the organic component is converted to $\mathrm{H}_{2} \mathrm{O}$ or $\mathrm{CO}_{2}$ in air. This conversion predominantly was observed at around $200{ }^{\circ} \mathrm{C}$ for OT ligands. From TEM images, the average sizes of OAm-OT and OT AuNPs were analyzed to be $4.9 \pm$ 0.4 and $4.4 \pm 0.4 \mathrm{~nm}$ in diameter, respectively. Combining the weight loss determined by TGA and the average size from TEM analysis, the ligand density of OAm-OT AuNPs and OT AuNPs was determined to be 4.5 and 4.7 ligands $/ \mathrm{nm}^{2}$ using a simplified spherical model ${ }^{58}$ (Table S1). The similarity in the ligand packing between the two synthesis routes suggests that the OAm method is an ideal alternative to the established onestep, one-phase method with distinct advantages. While the reaction temperature is an important parameter in the onephase method, size control over the full reported range typically 


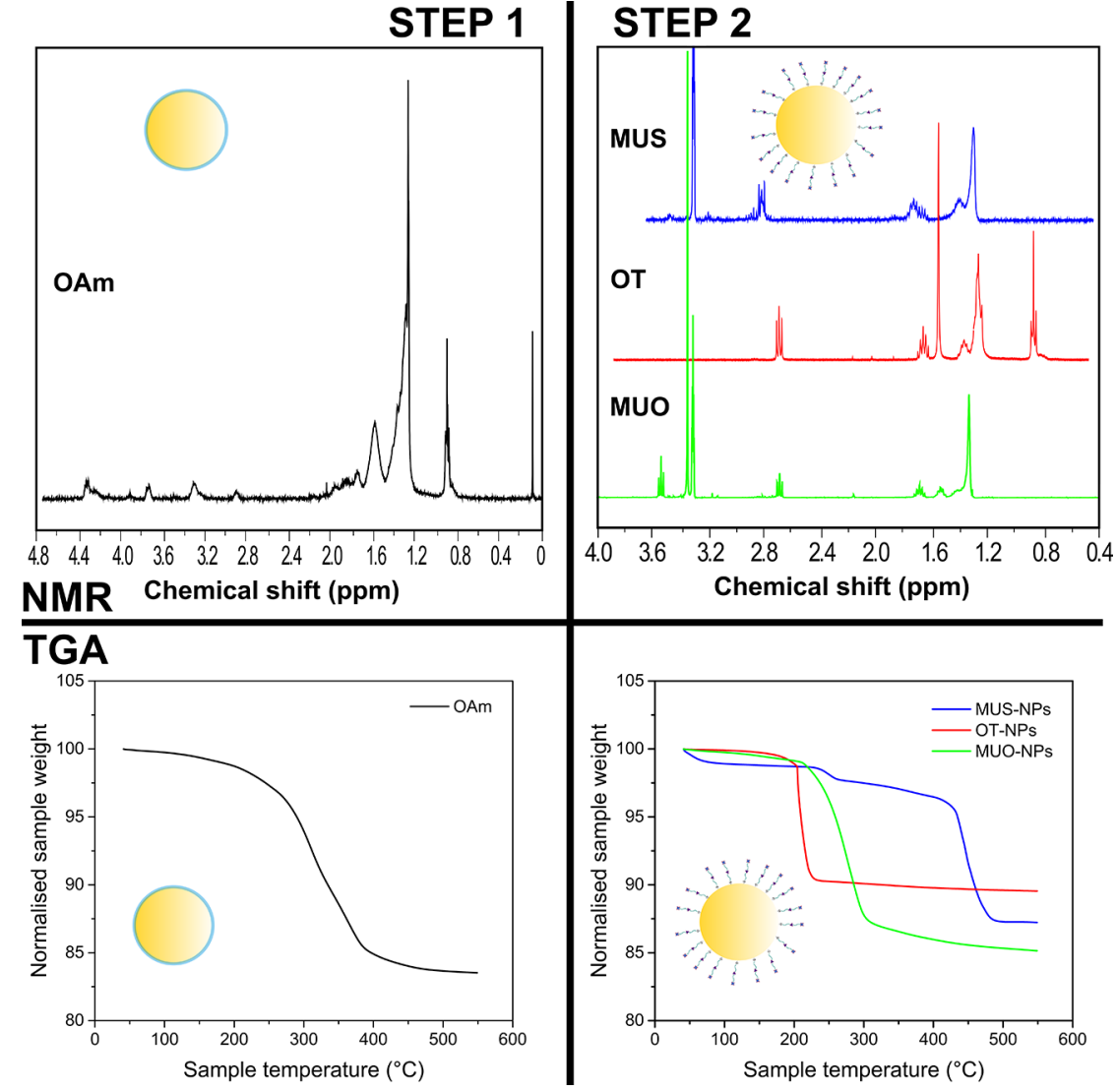

Figure 3. Thiol-for-OAm ligand exchange for homoligand AuNPs. NMR and TGA results for AuNPs before and after thiol-for-OAm ligand exchange using MUS, OT, and MUO, respectively. The ${ }^{1} \mathrm{H}$ NMR spectra of various AuNPs were obtained after purification and subsequent $\mathrm{I}_{2}-$ induced ligand desorption.

requires further adjustment of the reaction solvent mixture. ${ }^{25}$ More importantly, the type of thiol ligand plays an important role in the observed size distribution of the AuNP core, making it particularly difficult to independently vary both parameters in systematic studies. ${ }^{32}$

Variation of Thiol Capping and Resulting Solubility of AuNPs. Various thiol-capped AuNPs from an extensive library of DCM-soluble ligands were successfully prepared via the presented thiol-for-OAm ligand exchange approach. An overview of the studied ligands and the resulting AuNP solubility after functionalization is shown in Table 1. UV-vis absorption spectroscopy was used to evaluate the AuNP solvation by tracking their respective plasmon resonance peak (Figure S9). The simple but versatile ligand-exchange step enabled the functionalization of thiol-capped AuNPs that could be dissolved in solvents ranging from nonpolar (hexane, toluene, and chloroform) to polar aprotic (DCM) and polar protic solvents $\left(\mathrm{H}_{2} \mathrm{O}, \mathrm{MeOH}\right.$, and EtOH). Furthermore, the complete thiol replacement was also evidenced by the disappearance of the OAm characteristic footprint from ${ }^{1} \mathrm{H}$ NMR, as presented in Figure 3. An overview of the obtained yield for both AuNP syntheses in OAm (step 1) and thiol-for-OAm AuNP functionalization (step 2) is shown in the Supporting Information (Table S2). While a reaction yield of $94.9 \%$ was recovered for step 1, we obtained yields of 91.6 and $87.8 \%$ for subsequent functionalization with MUA and MUO, respectively.

AuNPs with various binary mixtures of thiol ligands were also studied by introducing a prescribed feed composition for OAm-
AuNPs with a size distribution of $4.9 \pm 0.4 \mathrm{~nm}$ during the thiolfor-OAm ligand exchange. The UV-vis results of different mixtures (Figure S11) indicate the solubility of MUS-OT AuNPs in $\mathrm{H}_{2} \mathrm{O}$, MUO-PET AuNPs in MeOH, MNT-HT AuNPs in chloroform, and MDDCBO-HT AuNPs in the liquid crystal (LC) 4-cyano-4'-pentylbiphenyl (5CB). Most distinct is the effect of a binary ligand mixture on solvation for a combination of MDDCBO and HT. While the homoligandprotected AuNPs with MDDCBO (or HT) showed very limited solubility in $5 \mathrm{CB}$, the binary ligand mixture of MDDCBO and HT greatly improved solvation. This can be explained by the introduction of the short ligand as a spacing agent. $^{42}$

Variation of Ligand Shell Composition for Binary Mixtures of MUS and OT. In order to establish a detailed relationship between the feed ratio of the binary ligand mixture in the thiol-for-OAm exchange step and the resulting ligand composition on the surface of the AuNPs, batches were prepared from six different molecular ratios of MUS and OT. The same OAm-protected AuNPs were used as based material throughout this study, with a size distribution of $3.6 \pm 0.5 \mathrm{~nm}$ in diameter. In detail, the washed and dried AuNPs after ligand exchange were dissolved in a mixture of $\mathrm{D}_{2} \mathrm{O}$ and $\mathrm{MeOD}$ (batches with MUS) and $\mathrm{CDCl}_{3}$ (the batch with OT only) for the purpose of AuNP (and $\mathrm{I}_{2}$ ) solvation. The ${ }^{1} \mathrm{H}$ NMR spectra of these solutions maintained only peaks from the solvents or impurities (Figures S12-S17), which suggests that a vast majority of the thiol ligands were attached to the AuNP surface. ${ }^{59}$ The addition of $\mathrm{I}_{2}$ in excess gave rise to the distinct 
and characteristic peaks by the desorption of the thiols from the AuNP surface. By comparing the integration data of peaks contributed by MUS and OT individually, the yield ratio was determined and compared with the original feed ratio, as presented in Figure $4 .^{57}$ Interestingly, the resulting surface
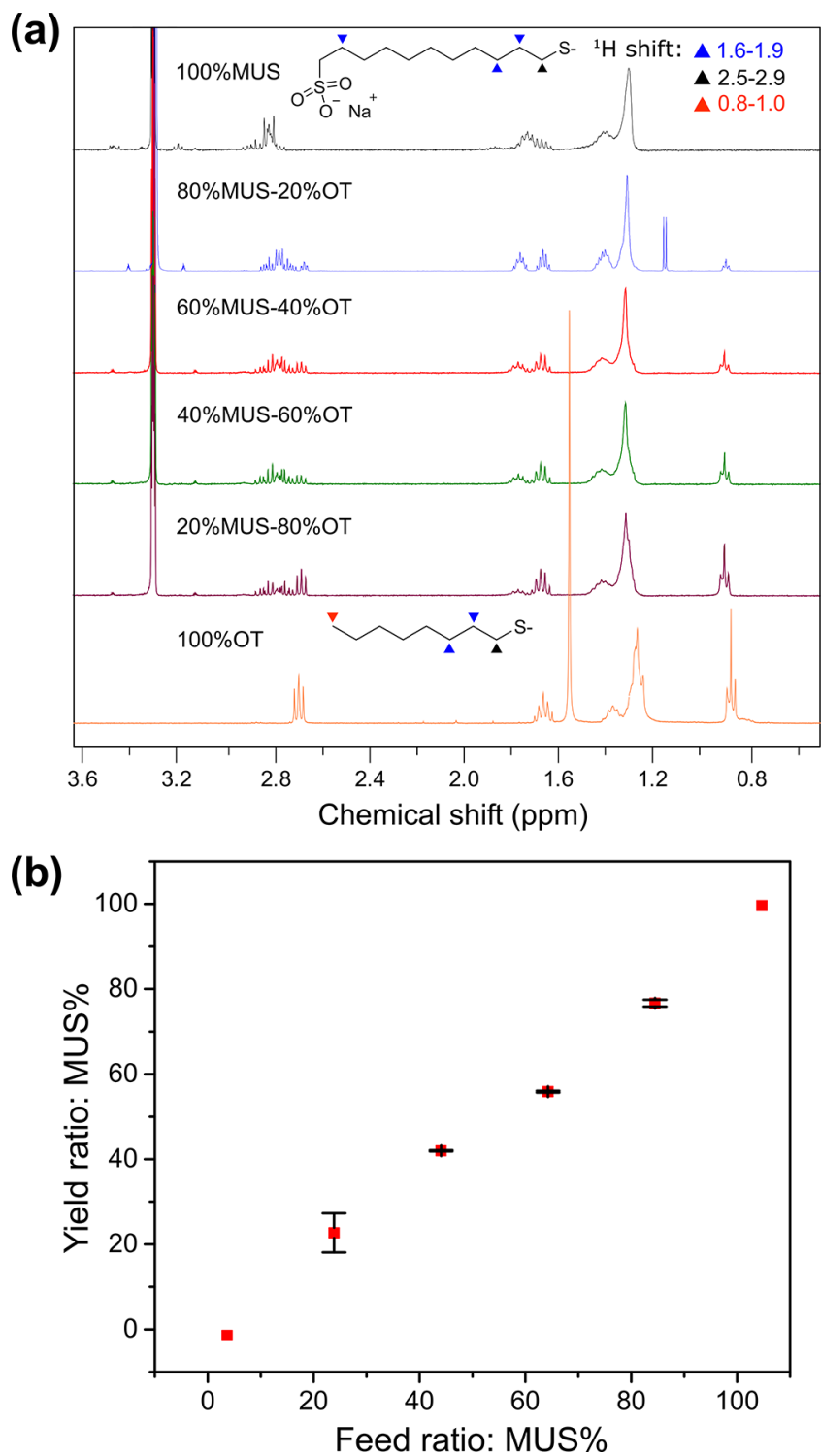

Figure 4. Variation of ligand composition for mixed ligand MUS-OT AuNPs through the feed ratio. (a) Chemical shift profile after purification and subsequent $\mathrm{I}_{2}$-induced ligand desorption and (b) the calculated ligand yield ratio with respect to the ligand feed ratio.

composition scaled accurately and reproducibly with the feed ratio variation, demonstrating that the ligand composition can be precisely controlled with the herein-presented approach.

These results are in stark contrast to thiol-for-thiol place exchange reactions, where the difference in thiol affinity for the gold surface poses a challenge for the consistent variation and tunability of the effective binary ligand composition on the AuNPs. ${ }^{60,61}$ In the present approach, the dominating competition for surface adsorption is between thiol and OAm rather than thiol and thiol. The composition variation was further supported by the TGA results shown in the Supporting Information (Figure S18). On the basis of the ligand shell molar ratio of MUS and OT obtained by NMR analysis, the partial packing density for each ligand component was calculated for the different MUS-OT batches, as shown in Table S3.

We note that for reliable and reproducible functionalization with a consistent relationship between the ligand feed in solution and ligand yield on the AuNPs, the presented route relies on the solubility of the respective target thiols in a common solvent with the OAm-protected AuNPs, here DCM. A vast library of thiol ligands are either hydrophobic or amphipathic and thus compatible with the approach. Indeed, most of the commonly used thiol ligands exhibit a hydrophobic alkyl backbone with a functional (and often polar) end group. In contrast, hydrophilic thiols with poor solubility in DCM require functionalization via a two-phase process with $\mathrm{H}_{2} \mathrm{O}$, which resulted in less precise control over the ligand composition. Additionally, the above shown tuning of the ligand shell composition by the feed ratio relies on a nonspecific coadsorption of both thiol ligands in a binary mixture. Recent work by Klajn and co-workers elucidated the effect of electrostatic interactions between different ligand types on the final composition of the ligand shell. ${ }^{55}$ For a mixture of a positively charged viologen-based ligand and a zwitterionic sulfobetaine ligand, a variation of the ligand feed ratio by a factor of 5000 changed the effective ligand composition on the nanoparticles by only a factor of 3 . In more general terms, we anticipate our approach to be a versatile nanoparticle platform for the study of both size and ligand composition on the resulting ligand shell morphology. ${ }^{62}$

\section{CONCLUSIONS}

The presented synthesis platform for AuNP synthesis enabled the decoupling of core size refinement and ligand shell variation in thiol-capped AuNPs. Following a modified synthesis with $\mathrm{OAm}$ as an intermediate protecting capping agent, the core size was tuned in the range of $2-7 \mathrm{~nm}$ by varying the reaction temperature. Subsequently, the functionalization of the OAmAuNPs with a wide choice of thiol ligands and binary mixtures thereof was achieved, resulting in tunable solubility ranging from $\mathrm{H}_{2} \mathrm{O}$ and alcohols to apolar organic solvents and LCs. Moreover, the thiol-for-OAm ligand exchange method proved to be as efficient as the conventional one-step method in preparing AuNPs with dense ligand packing. For MUS-OTprotected AuNPs, a consistent relationship was established between the thiol ligand mixture in the feed solution and the resulting composition on the AuNP, demonstrating precise control over the surface properties. We regard this synthesis platform as a valuable tool for fabricating thiol-protected AuNPs with rational design optimization for target applications.

\section{ASSOCIATED CONTENT}

\section{Supporting Information}

The Supporting Information is available free of charge on the ACS Publications website at DOI: 10.1021/acs.langmuir.8b00353.

TEM histograms of synthesized NPs; further experimental details; and NMR, TGA, MS, and UV-vis data (PDF)

\section{AUTHOR INFORMATION}

\section{Corresponding Author}

*E-mail: s.guldin@ucl.ac.uk. 


\section{ORCID}

Ye Yang: 0000-0001-9344-9349

Luis A. Serrano: 0000-0001-5292-5150

Stefan Guldin: 0000-0002-4413-5527

\section{Notes}

The authors declare no competing financial interest.

\section{ACKNOWLEDGMENTS}

This project received funding from the European Union's Horizon 2020 research and innovation programme under grant agreement no. 633635 (DIACHEMO). Y.Y. acknowledges University College London for the Overseas Research Scholarship and the Graduate Research Scholarship. S.G. is thankful for support by a start-up fund from the Department of Chemical Engineering at University College London. The authors are grateful to Dr. Paulo Jacob Silva and Prof. Francesco Stellacci (EPFL) for providing the MUS ligand and Dr. Jakub W. Trzcinski for valuable feedback on the manuscript.

\section{REFERENCES}

(1) Barnes, W. L.; Dereux, A.; Ebbesen, T. W. Surface Plasmon Subwavelength Optics. Nature 2003, 424, 824-830.

(2) Tian, Y.; Tatsuma, T. Mechanisms and Applications of PlasmonInduced Charge Separation at $\mathrm{TiO} 2$ Films Loaded with Gold Nanoparticles. J. Am. Chem. Soc. 2005, 127, 7632-7637.

(3) Haruta, M.; Yamada, N.; Kobayashi, T.; Iijima, S. Gold Catalysts Prepared by Coprecipitation for Low-Temperature Oxidation of Hydrogen and of Carbon Monoxide. J. Catal. 1989, 115, 301-309.

(4) Corma, A.; Garcia, H. Supported Gold Nanoparticles as Catalysts for Organic Reactions. Chem. Soc. Rev. 2008, 37, 2096-2126.

(5) Wang, C.; Yin, H.; Dai, S.; Sun, S. A General Approach to Noble Metal-Metal Oxide Dumbbell Nanoparticles and Their Catalytic Application for CO Oxidation. Chem. Mater. 2010, 22, 3277-3282.

(6) Howes, P. D.; Chandrawati, R.; Stevens, M. M. Colloidal Nanoparticles as Advanced Biological Sensors. Science 2014, 346, 1247390

(7) Dykman, L.; Khlebtsov, N. Gold Nanoparticles in Biomedical Applications: Recent Advances and Perspectives. Chem. Soc. Rev. 2012, 41, 2256-2282.

(8) Shukla, R.; Bansal, V.; Chaudhary, M.; Basu, A.; Bhonde, R. R.; Sastry, M. Biocompatibility of Gold Nanoparticles and Their Endocytotic Fate inside the Cellular Compartment: a Microscopic Overview. Langmuir 2005, 21, 10644-10654.

(9) Kinnear, C.; Moore, T. L.; Rodriguez-Lorenzo, L.; RothenRutishauser, B.; Petri-Fink, A. Form Follows Function: Nanoparticle Shape and Its Implications for Nanomedicine. Chem. Rev. 2017, 117, $11476-11521$.

(10) Castner, D. G.; Hinds, K.; Grainger, D. W. X-ray Photoelectron Spectroscopy Sulfur 2p Study of Organic Thiol and Disulfide Binding Interactions with Gold Surfaces. Langmuir 1996, 12, 5083-5086.

(11) Bürgi, T. Properties of the Gold-Sulphur Interface: from SelfAssembled Monolayers to Clusters. Nanoscale 2015, 7, 15553-15567.

(12) Giljohann, D. A.; Seferos, D. S.; Daniel, W. L.; Massich, M. D.; Patel, P. C.; Mirkin, C. A. Gold Nanoparticles for Biology and Medicine. Angew. Chem., Int. Ed. 2010, 49, 3280-3294.

(13) Saha, K.; Agasti, S. S.; Kim, C.; Li, X.; Rotello, V. M. Gold Nanoparticles in Chemical and Biological Sensing. Chem. Rev. 2012, 112, 2739-2779.

(14) Yeh, Y.-C.; Creran, B.; Rotello, V. M. Gold Nanoparticles: Preparation, Properties, and Applications in Bionanotechnology. Nanoscale 2012, 4, 1871-1880.

(15) Le Ouay, B.; Guldin, S.; Luo, Z.; Allegri, S.; Stellacci, F. Freestanding Ultrathin Nanoparticle Membranes Assembled at Transient Liquid-Liquid Interfaces. Adv. Mater. Interfaces 2016, 3, 1600191.
(16) DeVries, G. A.; Brunnbauer, M.; Hu, Y.; Jackson, A. M.; Long, B.; Neltner, B. T.; Uzun, O.; Wunsch, B. H.; Stellacci, F. Divalent Metal Nanoparticles. Science 2007, 315, 358-361.

(17) Kuna, J. J.; Voïtchovsky, K.; Singh, C.; Jiang, H.; Mwenifumbo, S.; Ghorai, P. K.; Stevens, M. M.; Glotzer, S. C.; Stellacci, F. The Effect of Nanometre-Scale Structure on Interfacial Energy. Nat. Mater. 2009, $8,837-842$.

(18) Colangelo, E.; Comenge, J.; Paramelle, D.; Volk, M.; Chen, Q.; Lévy, R. Characterizing Self-Assembled Monolayers on Gold Nanoparticles. Bioconjugate Chem. 2017, 28, 11-22.

(19) Bradford, S. M.; Fisher, E. A.; Meli, M.-V. Ligand Shell Composition-Dependent Effects on the Apparent Hydrophobicity and Film Behavior of Gold Nanoparticles at the Air-Water Interface. Langmuir 2016, 32, 9790-9796.

(20) Edwards, W.; Marro, N.; Turner, G.; Kay, E. R. Continuum Tuning of Nanoparticle Interfacial Properties by Dynamic Covalent Exchange. Chem. Sci. 2018, 9, 125-133.

(21) Jiang, Y.; Huo, S.; Mizuhara, T.; Das, R.; Lee, Y.-W.; Hou, S.; Moyano, D. F.; Duncan, B.; Liang, X.-J.; Rotello, V. M. The Interplay of Size and Surface Functionality on the Cellular Uptake of Sub-10 nm Gold Nanoparticles. ACS Nano 2015, 9, 9986-9993.

(22) Hung, A.; Mwenifumbo, S.; Mager, M.; Kuna, J. J.; Stellacci, F.; Yarovsky, I.; Stevens, M. M. Ordering Surfaces on the Nanoscale: Implications for Protein Adsorption. J. Am. Chem. Soc. 2011, 133, $1438-1450$

(23) Huang, R.; Carney, R. P.; Stellacci, F.; Lau, B. L. ProteinNanoparticle Interactions: the Effects of Surface Compositional and Structural Heterogeneity Are Scale Dependent. Nanoscale 2013, 5, 6928-6935.

(24) Brust, M.; Fink, J.; Bethell, D.; Schiffrin, D.; Kiely, C. Synthesis and Reactions of Functionalised Gold Nanoparticles. J. Chem. Soc., Chem. Commun. 1995, 1655-1656.

(25) Zheng, N.; Fan, J.; Stucky, G. D. One-Step One-Phase Synthesis of Monodisperse Noble-Metallic Nanoparticles and Their Colloidal Crystals. J. Am. Chem. Soc. 2006, 128, 6550-6551.

(26) Wu, B.-H.; Yang, H.-Y.; Huang, H.-Q.; Chen, G.-X.; Zheng, N.F. Solvent Effect on the Synthesis of Monodisperse Amine-Capped Au Nanoparticles. Chin. Chem. Lett. 2013, 24, 457-462.

(27) Shon, Y.-S.; Choo, H. Organic Reactions of MonolayerProtected Metal Nanoparticles. C. R. Chim. 2003, 6, 1009-1018.

(28) Neouze, M.-A.; Schubert, U. Surface Modification and Functionalization of Metal and Metal Oxide Nanoparticles by Organic Ligands. Monatsh. Chem. 2008, 139, 183-195.

(29) Hostetler, M. J.; Green, S. J.; Stokes, J. J.; Murray, R. W. Monolayers in Three Dimensions: Synthesis and Electrochemistry of $\omega$-Functionalized Alkanethiolate-Stabilized Gold Cluster Compounds. J. Am. Chem. Soc. 1996, 118, 4212-4213.

(30) Luo, Z.; Hou, J.; Menin, L.; Ong, Q. K.; Stellacci, F. Evolution of the Ligand Shell Morphology during Ligand Exchange Reactions on Gold Nanoparticles. Angew. Chem., Int. Ed. 2017, 56, 13521-13525.

(31) Hurst, S. J.; Lytton-Jean, A. K.; Mirkin, C. A. Maximizing DNA Loading on a Range of Gold Nanoparticle Sizes. Anal. Chem. 2006, 78, 8313-8318.

(32) Woehrle, G. H.; Brown, L. O.; Hutchison, J. E. ThiolFunctionalized, 1.5-nm Gold Nanoparticles through Ligand Exchange Reactions: Scope and Mechanism of Ligand Exchange. J. Am. Chem. Soc. 2005, 127, 2172-2183.

(33) Shichibu, Y.; Negishi, Y.; Tsukuda, T.; Teranishi, T. Large-Scale Synthesis of Thiolated Au25 Clusters via Ligand Exchange Reactions of Phosphine-Stabilized Au11 Clusters. J. Am. Chem. Soc. 2005, 127, 13464-13465.

(34) Gittins, D.; Caruso, F. Spontaneous Phase Transfer of Nanoparticulate Metals from Organic to Aqueous Media. Angew. Chem., Int. Ed. 2001, 40, 3001-3004.

(35) Rucareanu, S.; Gandubert, V. J.; Lennox, R. B. 4-(N,NDimethylamino)pyridine-Protected Au Nanoparticles: Versatile Precursors for Water- and Organic-Soluble Gold Nanoparticles. Chem. Mater. 2006, 18, 4674-4680. 
(36) Manea, F.; Bindoli, C.; Polizzi, S.; Lay, L.; Scrimin, P. Expeditious Synthesis of Water-Soluble, Monolayer-Protected Gold Nanoparticles of Controlled Size and Monolayer Composition. Langmuir 2008, 24, 4120-4124.

(37) Brinas, R. P.; Maetani, M.; Barchi, J. J., Jr. A Survey of PlaceExchange Reaction for the Preparation of Water-Soluble Gold Nanoparticles. J. Colloid Interface Sci. 2013, 392, 415-421.

(38) Wilcoxon, J. P.; Provencio, P. Etching and Aging Effects in Nanosize $\mathrm{Au}$ Clusters Investigated Using High-Resolution SizeExclusion Chromatography. J. Phys. Chem. B 2003, 107, 12949-12957.

(39) Shichibu, Y.; Negishi, Y.; Tsunoyama, H.; Kanehara, M.; Teranishi, T.; Tsukuda, T. Extremely High Stability of GlutathionateProtected Au25 Clusters Against Core Etching. Small 2007, 3, 835839.

(40) Baranov, D.; Kadnikova, E. N. Synthesis and Characterization of Azidoalkyl-Functionalized Gold Nanoparticles as Scaffolds for "Click"Chemistry Derivatization. J. Mater. Chem. 2011, 21, 6152-6157.

(41) Ojea-Jimenez, I.; Romero, F. M.; Bastus, N. G.; Puntes, V. Small Gold Nanoparticles Synthesized with Sodium Citrate and Heavy Water: Insights into the Reaction Mechanism. J. Phys. Chem. C 2010, 114, 1800-1804.

(42) Milette, J.; Toader, V.; Reven, L.; Lennox, R. B. Tuning the Miscibility of Gold Nanoparticles Dispersed in Liquid Crystals via the Thiol-for-DMAP Reaction. J. Mater. Chem. 2011, 21, 9043-9050.

(43) Isaacs, S.; Cutler, E.; Park, J.; Lee, T.; Shon, Y. Synthesis of Tetraoctylammonium-Protected Gold Nanoparticles with Improved Stability. Langmuir 2005, 21, 5689-5692.

(44) Zhao, P.; Li, N.; Astruc, D. State of the Art in Gold Nanoparticle Synthesis. Coord. Chem. Rev. 2013, 257, 638-665.

(45) Mourdikoudis, S.; Liz-Marzán, L. M. Oleylamine in Nanoparticle Synthesis. Chem. Mater. 2013, 25, 1465-1476.

(46) Hiramatsu, H.; Osterloh, F. E. A Simple Large-Scale Synthesis of Nearly Monodisperse Gold and Silver Nanoparticles with Adjustable Sizes and with Exchangeable Surfactants. Chem. Mater. 2004, 16, 2509-2511.

(47) Lau, C. Y.; Duan, H.; Wang, F.; He, C. B.; Low, H. Y.; Yang, J. K. Enhanced Ordering in Gold Nanoparticles Self-Assembly through Excess Free Ligands. Langmuir 2011, 27, 3355-3360.

(48) Ojea-Jiménez, I.; García-Fernández, L.; Lorenzo, J.; Puntes, V. F. Facile Preparation of Cationic Gold Nanoparticle-Bioconjugates for Cell Penetration and Nuclear Targeting. ACS Nano 2012, 6, 76927702

(49) Zhang, T.; Guerin, D.; Alibart, F.; Vuillaume, D.; Lmimouni, K.; Lenfant, S.; Yassin, A.; Oçafrain, M.; Blanchard, P.; Roncali, J. Negative Differential Resistance, Memory, and Reconfigurable Logic Functions Based on Monolayer Devices Derived from Gold Nanoparticles Functionalized with Electropolymerizable TEDOT Units. J. Phys. Chem. C 2017, 121, 10131-10139.

(50) Peng, S.; Lee, Y.; Wang, C.; Yin, H.; Dai, S.; Sun, S. A Facile Synthesis of Monodisperse Au Nanoparticles and Their Catalysis of CO Oxidation. Nano Res. 2008, 1, 229-234.

(51) Shen, C.; Hui, C.; Yang, T.; Xiao, C.; Tian, J.; Bao, L.; Chen, S.; Ding, H.; Gao, H. Monodisperse Noble-Metal Nanoparticles and Their Surface Enhanced Raman Scattering Properties. Chem. Mater. 2008, 20, 6939-6944.

(52) Kluenker, M.; Mondeshki, M.; Nawaz Tahir, M.; Tremel, W. Monitoring Thiol-Ligand Exchange on $\mathrm{Au}$ Nanoparticle Surfaces. Langmuir 2018, 34, 1700-1710.

(53) Malicki, M.; Hales, J. M.; Rumi, M.; Barlow, S.; McClary, L.; Marder, S. R.; Perry, J. W. Excited-State Dynamics and Dye-Dye Interactions in Dye-Coated Gold Nanoparticles with Varying Alkyl Spacer Lengths. Phys. Chem. Chem. Phys. 2010, 12, 6267-6277.

(54) Köhntopp, A.; Dabrowski, A.; Malicki, M.; Temps, F. Photoisomerisation and Ligand-Controlled Reversible Aggregation of Azobenzene-Functionalised Gold Nanoparticles. Chem. Commun. 2014, 50, 10105-10107.

(55) Chu, Z.; Han, Y.; Kral, P.; Klajn, R. "Precipitation on Nanoparticles": Attractive Intermolecular Interactions Stabilize
Specific Ligand Ratios on the Surfaces of Nanoparticles. Angew. Chem., Int. Ed. 2018, 57, 1-6.

(56) Liu, X.; Hu, Y.; Stellacci, F. Mixed-Ligand Nanoparticles as Supramolecular Receptors. Small 2011, 7, 1961-1966.

(57) van Lehn, R. C.; Ricci, M.; Silva, P. H.; Andreozzi, P.; Reguera, J.; Voitchovsky, K.; Stellacci, F.; Alexander-Katz, A. Lipid Tail Protrusions Mediate the Insertion of Nanoparticles into Model Cell Membranes. Nat. Commun. 2014, 5, 4482.

(58) Harkness, K. M.; Hixson, B. C.; Fenn, L. S.; Turner, B. N.; Rape, A. C.; Simpson, C. A.; Huffman, B. J.; Okoli, T. C.; McLean, J. A.; Cliffel, D. E. A Structural Mass Spectrometry Strategy for the Relative Quantitation of Ligands on Mixed Monolayer-Protected Gold Nanoparticles. Anal. Chem. 2010, 82, 9268-9274.

(59) Badia, A.; Gao, W.; Singh, S.; Demers, L.; Cuccia, L.; Reven, L. Structure and chain dynamics of alkanethiol-capped gold colloids. Langmuir 1996, 12, 1262-1269.

(60) Smith, A. M.; Marbella, L. E.; Johnston, K. A.; Hartmann, M. J.; Crawford, S. E.; Kozycz, L. M.; Seferos, D. S.; Millstone, J. E. Quantitative Analysis of Thiolated Ligand Exchange on Gold Nanoparticles Monitored by $1 \mathrm{H}$ NMR Spectroscopy. Anal. Chem. 2015, 87, 2771-2778.

(61) Goldmann, C.; Ribot, F.; Peiretti, L. F.; Quaino, P.; Tielens, F.; Sanchez, C.; Chanéac, C.; Portehault, D. Quantified Binding Scale of Competing Ligands at the Surface of Gold Nanoparticles: The Role of Entropy and Intermolecular Forces. Small 2017, 13, 1604028.

(62) Luo, Z.; Marson, D.; Ong, Q. K.; Loiudice, A.; Kohlbrecher, J.; Radulescu, A.; Krause-Heuer, A.; Darwish, T.; Balog, S.; Buonsanti, R.; Svergun, D. I.; Posocco, P.; Stellacci, F. Quantitative 3D Determination of Self-Assembled Structures on Nanoparticles Using Small Angle Neutron Scattering. Nat. Commun. 2018, 9, 1343. 